\title{
INVESTIGATION OF TWO MODELS OF TRINITROBENZENESULFONIC ACID-INDUCED COLITIS IN RATS
}

\author{
V. Marinov ${ }^{1 *}$, M. Eftimov ${ }^{1}$, M. Tzaneva ${ }^{2}$, M. Zhelyazkova ${ }^{1}$, S. Valcheva-Kuzmanova ${ }^{1}$ \\ ${ }^{1}$ Department of Preclinical and Clinical Pharmacology, Medical University Prof. Dr. Paraskev \\ Stoyanov, Varna, Bulgaria \\ ${ }^{2}$ Department of Preclinical and Clinical Sciences, Medical University Prof. Dr. Paraskev Stoyanov, \\ Varna Bulgaria
}

\begin{abstract}
PURPOSE: The aim of this study was to comparatively evaluate rat experimental colitis resulting from administration of two different doses of trinitrobenzenesulfonic acid (TNBS) dissolved in different volumes of two ethanol concentrations. METHODS: TNBS was applied in the colon by a soft cannula at a depth of about $10 \mathrm{~cm}$ from anus. The first group (TNBS20) was treated with $20 \mathrm{mg}$ TNBS dissolved in 0.4 $\mathrm{ml}$ of $40 \%$ ethanol solution. The second group (TNBS10) was treated with $10 \mathrm{mg}$ TNBS dissolved in 0.25 $\mathrm{ml}$ of $50 \%$ ethanol solution. The animals were sacrificed 24 hours after the treatment and the colon was examined macroscopically and histopathologically. RESULTS: There were no significant differences regarding the degree of hyperemia, total area of injury and thickening of the colon wall. The higher TNBS dose produced significantly more pronounced shortening of the colon and larger necrotic area. Histopathological evaluation showed similar degree of injuries in the two models. CONCLUSION: The two investigated models of TNBS-induced colitis were comparable according to the overall macroscopic and microscopic criteria for colitis evaluation.
\end{abstract}

Key words: Wistar rats, TNBS, models of colitis, IBD

\section{INTRODUCTION}

Inflammatory bowel disease (IBD) includes Crohn's disease and ulcerative colitis - two forms of inflammatory conditions of the colon and small intestine. Both forms usually involve severe diarrhea, pain and weight loss. IBD can be debilitating and sometimes leads to lifethreatening complications. IBD falls into the class of autoimmune diseases, in which the body's own immune system attacks elements of the digestive system. The treatement of the disease is difficult and incomplete and generally immunosuppression is required. The ethiology and pathogenesis are not completely explored to date and have been the object of extensive research recently. Animal models are used in which a chemical agent induces injuries to the colon similar to that of the actual disease. Trinitrobenzenesulfonic acid (TNBS)

\footnotetext{
*Correspondence to: assist. prof. Veselin Marinov, Department of Preclinical and Clinical Pharmacology, Medical University Prof. Dr. Paraskev Stoyanov, 9002 Varna, 55 M. Drinov Str., Bulgaria; Tel. 0899818886; E-mail: veselin.marinov@mu-varna.bg
}

is a chemical hapten that binds to tissue proteins and is capable of stimulating cellmediated immunity (1). TNBS is an agent commonly used to induce experimental models of colitis. There are several different variations in the model implementation.

The aim of the present study was to compare two versions of the experimental models of TNBS-induced colitis, resulting from administration of two different doses of TNBS dissolved in different volumes of two different ethanol concentrations

\section{MATERIALS AND METHODS Experimental substances}

Trinitrobenzene sulfonic acid (Sigma Aldrich, Germany) was used in the experiment.

\section{Animals and treatment}

Male Wistar rats (290-340 g) were used. The animals were housed in plastic cages in a wellventilated room maintained at $22 \pm 1^{\circ} \mathrm{C}$ and on a $12 / 12$ light/dark cycle. They had free access to food and drinking water. All procedures concerning animal treatment and experimentation were conducted in compliance 
MARINOV V., et al.

with the National and International laws and policies (EEC Council Directive 86/609).

The experimental animals were divided into two groups of 10 animals each. Rats were fasted for 24 hours. Before TNBS administration, animals were anesthesized with thiopental intraperitoneally to provide a painless procedure. The TNBS solution was applied in the colon through the rectum by a soft cannula inserted at a depth of about $10 \mathrm{~cm}$ from anus. The first group (TNBS20) was treated with $20 \mathrm{mg}$ TNBS dissolved in $0.4 \mathrm{ml}$ of $40 \%$ ethanol solution. The second group (TNBS10) was treated with $10 \mathrm{mg}$ TNBS dissolved in $0.25 \mathrm{ml}$ of $50 \%$ ethanol solution. After TNBS administration, the rats were left in a Trendelenburg position for several minutes to prevent leakage from the anus. The animals were sacrificed 24 hours after the procedure. The colon was excised up to the ileum, washed and opened to be evaluated macroscopically. Tissues were imbedded in $10 \%$ neutral buffered formalin solution for histopathological examination.

\section{Macroscopic evaluation}

Macroscopic changes were evaluated on the basis of criteria (2) given in Table 1.

Table 1. Scoring criteria applied to the visible macroscopic lesions in the rat colon

\begin{tabular}{ll}
\hline Criterion & Points assigned \\
\hline Adhesions & $0-$ No adhesions \\
& $1-$ Difficult dissection \\
& $2-$ Visible adhesions \\
& $3-$ "Wrapped" intestine \\
Colon length in cm & \\
Area of injury in $\mathrm{cm}^{2}$ & $0-$ Similar to uninflamed intestine \\
Thickening of the wall & $1-$ Thicker than normal $(\sim 1-2$ mm) \\
& $2-$ Much thicker than normal (>2 mm) \\
& $0-$ Similar to uninflamed intestine \\
& $1-$ Mild and generalized or intense but localized hyperemia \\
Hyperemia & $2-$ Intense and generalized hyperemia \\
& $3-$ Frank hemorrhage \\
& $0-$ No signs of necrosis \\
& $1-$ Small areas of necrosis \\
& $2-$ Patchy necrosis (cobblestone appearance) \\
& $3-$ Focal necrosis, $\oslash<0.8 \mathrm{~cm}$ \\
& $4-$ Focal necrosis, $\oslash>0.8 \mathrm{~cm}$ \\
& $5-$ Extended necrotic lesion \\
\hline
\end{tabular}

\section{Microscopic evaluation}

Colon tissues were individually fixed in $10 \%$ formalin, dehydrated, paraffin embedded, processed and stained with haematoxylin and eosin (H\&E).

\section{Statistical analysis}

Student's $t$-test was used to compare variables characterizing the two groups. A level of $\mathrm{p}<$
0.05 was considered significant. GraphPad Prism statistical software was used.

\section{RESULTS}

The results from the macroscopic evaluation of the colon tissue of the two groups are presented in Table 2.

Table 2. Scores of the visible macroscopic lesions in the rat colon for groups TNBS20 and TNBS1

\begin{tabular}{lll}
\hline Criteria & TNBS20 & TNBS10 \\
\hline Adhesions & 0 & 0 \\
Area of injury in $\mathrm{cm}^{2}$ & $6.063 \pm 1.219$ & $7.505 \pm 0.9136$ \\
Thickening & $1.875 \pm 0.1250$ & $1.900 \pm 0.1000$ \\
Hyperemia & $2.000 \pm 0.1890$ & $2.300 \pm 0.1528$ \\
Necrosis & $4.750 \pm 0.1637$ & $4.000 \pm 0.2582$ \\
\hline
\end{tabular}


A typical macroscopic view of rat colon from group TNBS20 is shown on Figure 1 and from group TNBS10 on Figure 2.

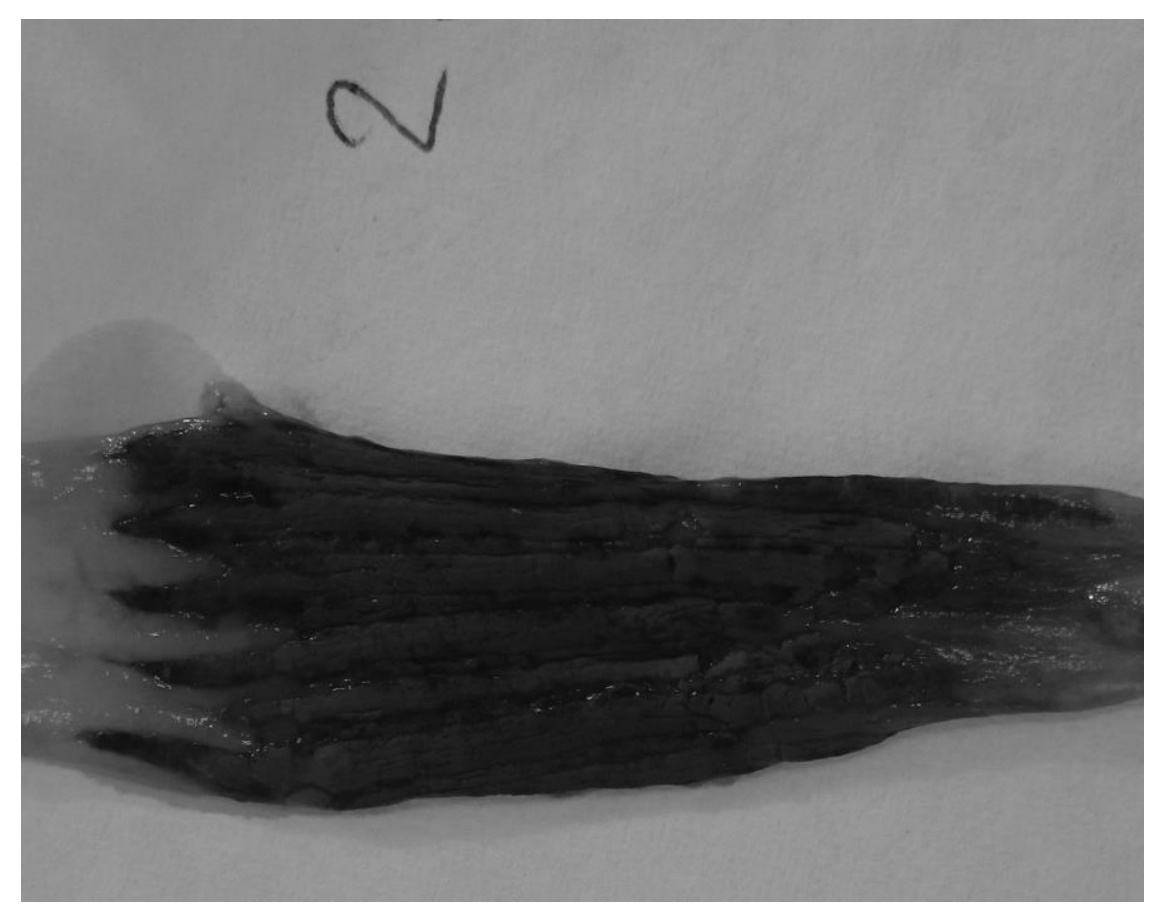

Figure 1. Macroscopic view of colon injury of a rat from group TNBS20

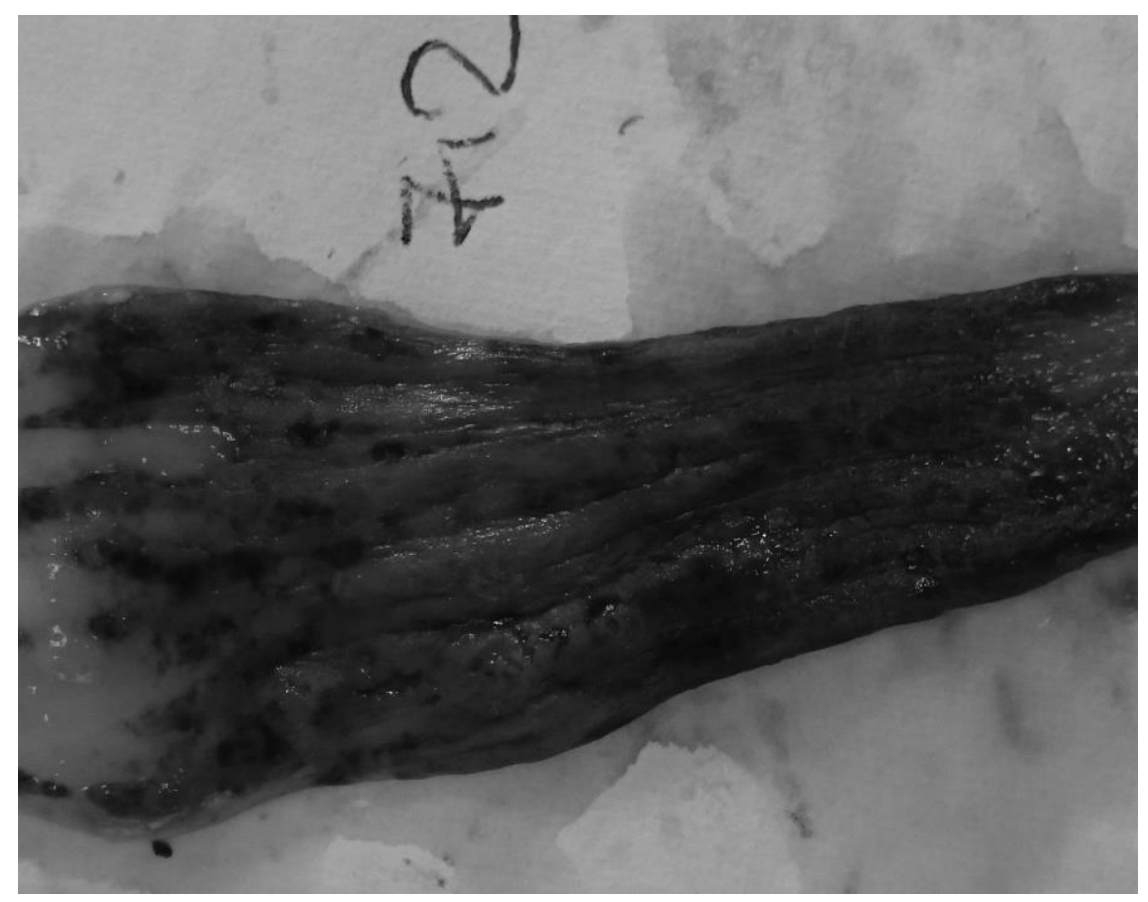

Figure 2. Macroscopic view of colon injury of a rat from group TNBS10

The length of the colon was significantly reduced by TNBS at a dose of $20 \mathrm{mg}$ in comparison with the dose of $10 \mathrm{mg}$ (Figure 3). The area of injury in TNBS10 group was slightly (but insignificantly) larger than that of TNBS20 group. There was no significant difference between TNBS20 and TNBS10 regarding thickening of the intestinal wall. Hyperemia was insignificantly increased in TNBS10 group compared to TNBS20 group. TNBS at a dose of $20 \mathrm{mg}$ caused a significantly more severe necrosis in comparison to TNBS at dose $10 \mathrm{mg}$ (Figure 4). 


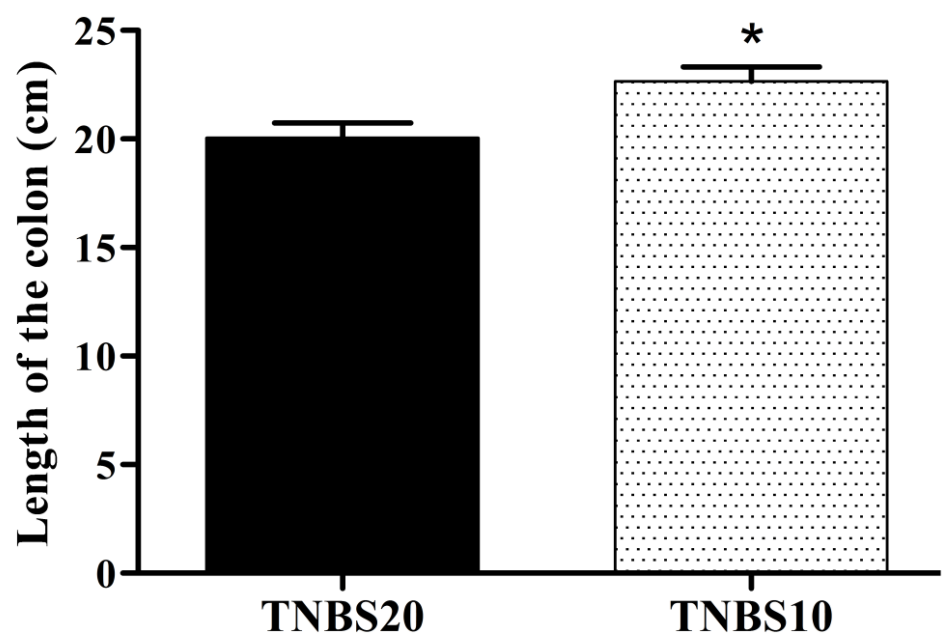

MARINOV V., et al.

Figure 3. Length of the colon

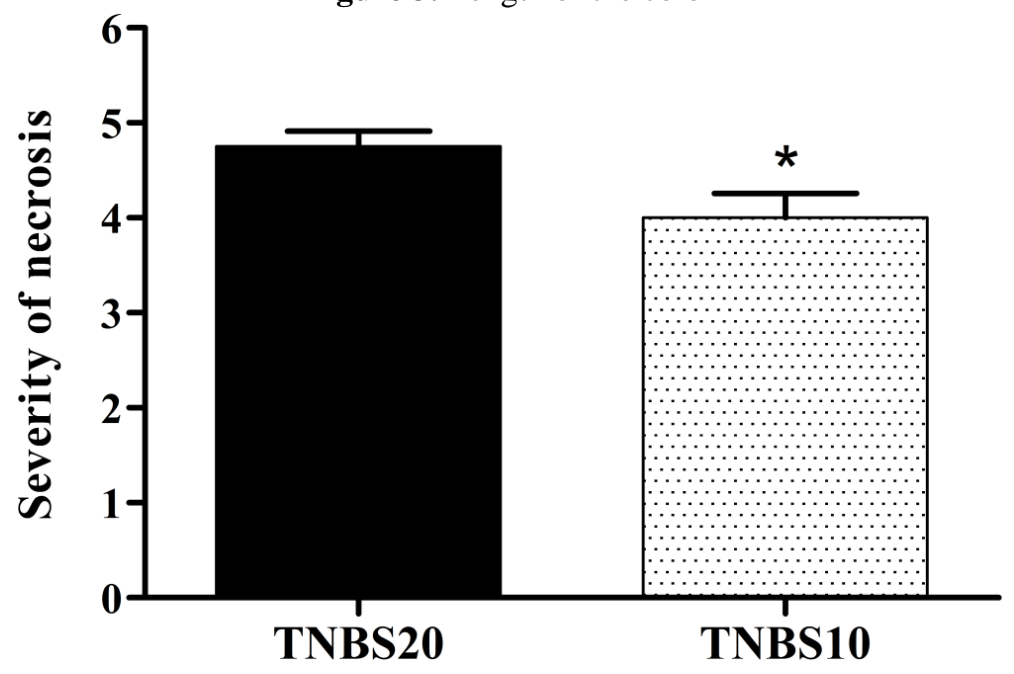

Figure 4. Severity of the necrosis

\section{Microscopic evaluation}

In the damaged areas histopathological evaluation showed diffuse mucosal ulceration involving the submucosa. Extensive and severe edema was observed. Some of the small blood vessels in the submucosa showed fibrinoid necrosis. Severe and diffuse inflammatory infiltration was present reaching the submucosa and involving the muscularis propria and the adipose tissue. The microscopic appearance of the injuries in the two models was similar. An example of the microscopic lesions from group TNBS20 is given in Figure 5, and one from group TNBS10 is shown in Figure 6.

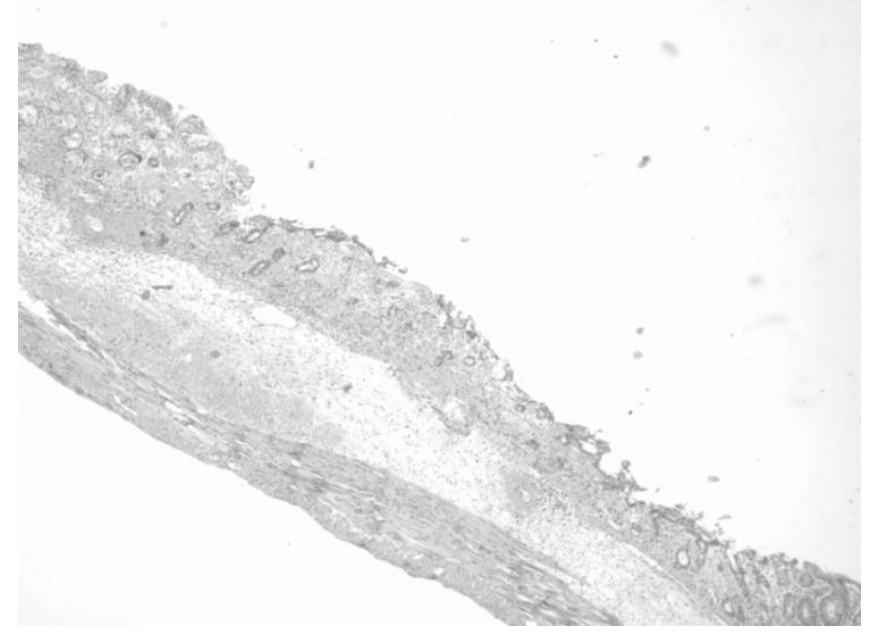

Figure 5. Microscopic view of a rat colon from group TNBS20. Degenerative changes in the epithelium, inflammatory infiltration, edema and hemorrhage in the submucosa (H\&E x 100) 


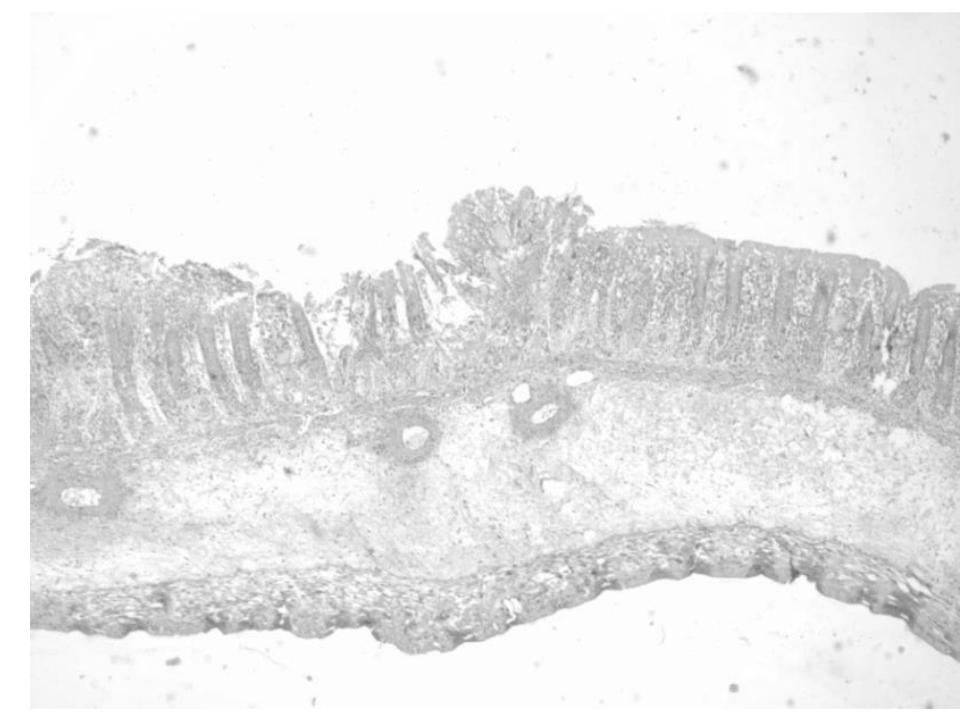

Figure 6. Microscopic view of a rat colon from group TNBS10. Erosions of the epithelium, destruction of the glandular layer and inflammatory changes, submucosal edema and fibrinoid necrosis of blood vessels (H\&E x 100)

\section{DISCUSSION}

Many authors have described the model of TNBS-induced colitis, but there are significant variations in the specific implementation of the method. The aim of the present work was to compare two combinations of different TNBS doses, volumes of introduced solution and concentration of the ethanol used as a solvent.

TNBS is an extremely potent oxidative agent. Oxidative stress has been implicated as a key factor in the pathogenesis of IBD both in human beings and experimental animals. One of the postulated mechanisms, whereby TNBS induces experimental colitis is through increased production of toxic reactive oxygen species (3).

Another important mechanism is the immunological one. When applied intrarectaly, TNBS acts as a chemical hapten to bind proteins in the colon wall and to stimulate cellmediated immunity (1). Delayed hypersensitivity reactions can be induced because it haptenates proteins with trinitrophenyl groups and renders such proteins immunogenic. This leads to a massive mucosal inflammation characterized by the dense infiltration of $\mathrm{T}$ cells and macrophages throughout the entire wall of the large bowel $(4, \quad 5)$. Delayed-type hypersensitivity is characterized by $\mathrm{T}$ cell-driven antigen-specific inflammatory cell infiltration within 12-72 hours after a second encounter with the antigen (6). Although the exact pathogenesis of IBD still remains unknown, several pathophysiological and histological features of IBD resemble hypersensitivity-like responses in the gastrointestinal tract $(7,8)$.

The dose of TNBS capable of inducing adequate inflammation has been reported to be between 50 and $150 \mathrm{mg} / \mathrm{kg}$. Doses above 150 $\mathrm{mg} / \mathrm{kg}$ are associated with mortality among the experimental animals (3). The doses used in this experiment are in the lowest end of this range. They roughly correspond to doses of 60 and $30 \mathrm{mg} / \mathrm{kg}$, respectively. It was obvious, that both doses worked similarly.

According to literature data, the volume of the inserted TNBS solution into the rectum varies from $0.25 \mathrm{ml}$ to $0.8 \mathrm{ml}$. Smaller volumes ensure remaining of the agent at the site of application and prevent leakage from the anus. We chose to use a relatively small volume $0.4 \mathrm{ml}$ in the first group and $0.25 \mathrm{ml}$ in the second. Nevertheless, we observed some features of leakage.

Ethanol may not be required for TNBSinduced damage, but this vehicle enhances the biological action of TNBS presumably by acting as a „barrier breaker“ (3). Higher ethanol concentrations of TNBS solution are supposed to have stronger damaging effect to the colon wall. This was exactly what we observed in our experiment. Most probably this was the main factor contributing to the absence of significant differences between the two TNBS-induced colitis models.

In conclusion, the results from this experiment demonstrate that TNBS at the two tested doses of $20 \mathrm{mg}$ and $10 \mathrm{mg}$ induced a pronounced experimental colitis. The effect of the smaller dose dissolved in 50\% ethanol at a volume of $0.25 \mathrm{ml}$ is almost equivalent to the effect of the higher dose dissolved in $40 \%$ ethanol at a volume of $0.40 \mathrm{ml}$. 


\section{REFERENCES}

1. Kim, H.S., Berstad, A., Experimental colitis in animal models. Scand J Gastroenterol, 27:529 537, 1992.

2. Daddaoua, A., Puerta, V., Zarzuelo, A. Suárez, M.D., Sánchez de Medina, F., Martínez-Augustin, O., Bovine Glycomacropeptide Is Anti-Inflammatory in Rats with Hapten-Induced Colitis. $J$ Nutr, 135:1164-1170, 2005.

3. Motavallian-Naeini, A., Andalib, S., Rabbani, M., Mahzouni, P., Afsharipour, M. and Minaiyan, M., Validation and optimization of experimental colitis induction in rats using $2,4,6-$ trinitrobenzene sulfonic acid. Res Pharm Sci; 7(3):159-169, 2012.

4. Li, J., Zhou, R., He, W.C., Xia, B., Effects of recombinant human intestinal trefoil factor on trinitrobenzene sulphonic acid
MARINOV V., et al. induced colitis in rats. Mol Biol Rep, 38(7):4787-4792, 2011.

5. Liu, X., Wang, J., Anti-inflammatory effects of iridoid glycosides fraction of Folium syringae leaves on TNBS-induced colitis in rats. $J$ Ethnopharmacol, 133(2):780-787, 2011.

6. Black, C. A., Delayed type hypersensitivity: current theories with an historic perspective. Dermatol Online J, 5:7, 1999.

7. Kraneveld, A. D., Buckley, T. L., van Heuven-Nolsen, D., van Schaik, Y., Koster, A. S., Nijkamp, F. P., Delayed-type hypersensitivity-induced increase in vascular permeability in the mouse small intestine: inhibition by depletion of sensory neuropeptides and NK1 receptor blockade. Br J Pharmacol, 114:1483-1489, 1995.

8. Lakatos, L., Immunology of inflammatory bowel diseases. Acta Physiol Hung, 87:355372, 2000. 